\title{
Prognostic Value of Neutrophil Lymphocyte Ratio in Second Line Advanced Malignant Pleural Mesothelioma
}

\author{
Ahmed El Bastawisy ${ }^{1}$, Maha Yahia1, Abdel Rahman M. Abdel Rahman², Rabab Gaafar1 \\ ${ }^{1}$ Medical Oncology Department, National Cancer Institute, Cairo University, Cairo, Egypt \\ ${ }^{2}$ Surgical Oncology Department, National Cancer Institute, Cairo University, Cairo, Egypt \\ Email: ${ }^{*}$ a_s basta@hotmail.com
}

Received 26 March 2014; revised 25 April 2014; accepted 20 May 2014

Copyright $(02014$ by authors and Scientific Research Publishing Inc.

This work is licensed under the Creative Commons Attribution International License (CC BY). http://creativecommons.org/licenses/by/4.0/

(c) (9) Open Access

\section{Abstract}

Background: Malignant pleural mesothelioma is a lethal disease and hence the strong need for identifying new prognostic factors. Methods: This is a retrospective study including all eligible patients with advanced malignant pleural mesothelioma (MPM) presenting to National Cancer Institute, Cairo University. Neutrophil lymphocyte (N/L) ratio was assessed before second line chemotherapy. 2.5 was used as the cutoff point. Endpoints were the assessment of correlation between N/L ratio and clinical response (CR), progression free survival (PFS) and overall survival (OS). Results: 52 patients (19 stage III and 33 stage IV) MPM were included and followed up during the period from July 2009 till November 2012 with a median follow-up period of 2.6 months. 87.5\% of patients with $\mathrm{N} / \mathrm{L}$ ratio $>2.5$ showed progressive disease versus $91.7 \%$ in patients with $\mathrm{N} / \mathrm{L}$ ratio < 2.5 (P-value = 0.66). 6-month PFS was $11 \%$ for patients with $\mathrm{N} / \mathrm{L}$ ratio $>2.5$ versus $14 \%$ for patients with $\mathrm{N} / \mathrm{L}$ ratio $<2.5(\mathrm{P}$-value $=0.001) .6$-month $\mathrm{OS}$ was $72 \%$ for patients with $\mathrm{N} / \mathrm{L}$ ratio $>2.5$ versus $66 \%$ for patients with $\mathrm{N} / \mathrm{L}$ ratio $<2.5(\mathrm{P}$-value $=0.4)$. Conclusion: $\mathrm{N} / \mathrm{L}$ ratio is a potential prognostic marker for advanced MPM treated with second line chemotherapy.

\section{Keywords}

Neutrophil Lymphocyte Ratio, Mesothelioma

\section{Introduction}

Malignant pleural mesothelioma is a rare disease with estimated incidence of 2500 cases in the United States

\footnotetext{
${ }^{*}$ Corresponding author.
}

How to cite this paper: El Bastawisy, A., Yahia, M., Rahman, A.R.M.A. and Gaafar, R. (2014) Prognostic Value of Neutrophil Lymphocyte Ratio in Second Line Advanced Malignant Pleural Mesothelioma. Journal of Cancer Therapy, 5, 717-722. 
every year [1]. The incidence is expected to rise within the next 20 years [2]. Occupational exposure to asbestos is a well-established etiological factor in $70 \%-80 \%$ of patients [2]. There are two famous scoring systems in malignant pleural mesothelioma. The first one is European Organization for Research and Treatment of Cancer (EORTC) scoring system including performance status, white blood cell (WBC) count, gender, histologic subtype, and whether the histologic diagnosis was definitive or not [3]. The second one is the Cancer and Leukemia Group B (CALGB) scoring system including performance status, age, WBC count, hemoglobin, presence or absence of weight loss, and chest pain [4]. However the complexity of these scoring systems raises concerns about the need for a more practical prognostic tool like neutrophil lymphocyte $(\mathrm{N} / \mathrm{L})$ ratio from a simple blood test. Neutrophil to lymphocyte ratio is used as a marker of subclinical inflammation. It is calculated by dividing the number of neutrophils by the number of lymphocytes, usually from peripheral blood sample [5] but sometimes also from cells that infiltrate tissue, such as tumor [6]. Increased N/L ratio is associated with poor prognosis of various cancers [7]. Inflammation is one of the postulated underlying mechanisms involved in occurrence and progression for several types of malignancies including malignant pleural mesothelioma in which asbestos fibers induced chronic tissue damage will produce local inflammatory reaction [8].

\section{Aim of Work}

The endpoints were assessment of correlation between N/L ratio and clinical response (CR), progression free survival (PFS) and overall survival (OS) in patients with second line advanced malignant pleural mesothelioma after second line systemic treatment.

\section{Methods}

This was a retrospective study including all eligible patients with advanced malignant pleural mesothelioma (MPM) presenting to National Cancer Institute, Cairo University where patients' files were reviewed for the following:

\subsection{Inclusion Criteria}

- The patient must have had a histologically confirmed diagnosis of malignant pleural mesothelioma.

- Recurrent stages III and IV MPM.

- Patient is at least 18 years of age.

- Patient with a performance status of (ECOG Scale) $\leq 2$.

- Patient with adequate bone marrow function, (WBC count $\geq 3.0 \times 10^{9} / \mathrm{L}, \mathrm{ANC} \geq 1.5 \times 10^{9} / \mathrm{L}$, platelet count $\geq 100 \times 10^{9} / \mathrm{L}$, hemoglobin level $\geq 9 \mathrm{~g} / \mathrm{L}$.

- Patient with adequate liver function; serum bilirubin $<1.5 \times$ ULN, ALT and AST levels $<$ three times normal values; ALT and AST levels < five times normal limits allowed in patients with known liver metastases.

- Patient with adequate Kidney function; plasma creatinine level $<1.5$ times normal value.

- Patients with a compliance, mental state and geographic proximity that allow adequate follow-up and they had to provide written informed consent before any study-specific procedure.

\subsection{Exclusion Criteria}

Patients who were pregnant or breastfeeding.

Patients with active second malignancy at the time of the study.

Patients who were involved in another clinical trial at the time of the study.

The study was conducted according to the Declaration of Helsinki and the guidelines for Good Clinical Practice. The local ethics committees approved the protocol.

\subsection{Treatment Plan}

Patients received one of the following two regimens:

1) Vinorelbine $25 \mathrm{mg} / \mathrm{m}^{2}$, IV on $100 \mathrm{cc}$ normal saline over 6 - 10 minutes followed by 100 normal saline IV D1, 8. Every three weeks, up to six cycles in responding patients.

2) Vorinostat (investigational) $300 \mathrm{mg}$ (or matching placebo) twice daily orally for 3 days each week of a 21-day cycle until progression or toxicity. 


\subsection{Study Assessment}

Pretreatment assessment included complete medical history and physical examination. Further assessment conducted within seven days before treatment included vital signs, performance status (ECOG), and complete blood count with differential and full biochemical panel, including liver and renal function tests were performed and repeated before each treatment course. Neutrophil lymphocyte (N/L) ratio was calculated by dividing the number of neutrophils by number of lymphocytes, from peripheral blood sample assessed before second line chemotherapy. 2.5 was used as the cutoff point. Radiological evaluation was carried out, including a computerized tomography (CT) scan of the chest and upper abdomen. Additional radiological imaging such as bone scan was done if indicated. Imaging was repeated every six weeks.

Evaluation was carried out according to modified RECIST criteria every six weeks [9].

Post-treatment evaluation included:

- Medical history and physical examination every three weeks.

- $\mathrm{CBC}$ and chemistry every three weeks.

- CT chest and upper abdomen every six weeks.

- Other investigations were done if indicated.

\subsection{Statistical Methods}

The SPSS package (version 17.0) was used for data analysis. Mean and standard deviation were reported to describe quantitative data. The chi-square and Fischer exact tests were used to evaluate the differences in the distribution of the variables. The Kaplan-Meier method was used to estimate the overall and PFS and the log rank test to evaluate differences in survival among groups. P-value of $\leq 0.05$ was considered significant.

\section{Results}

Table 1 summarizes patients' characteristics with regard to age, sex, smoking history, asbestos exposure history, histology, ECOG PS, stage and type of second line chemotherapy.

\section{Clinical Response:}

There was no statistically significant difference in clinical response based on N/L ratio where $91.7 \%$ of patients with $\mathrm{N} / \mathrm{L}$ ratio $<2.5$ showed $\mathrm{PD}$ as compared to $87.5 \%$ of patients with ratio $>2.5$. P-value $=0.66$ (Table 2).

PFS:

1) There was a statistically significant lower six months PFS in patients with $N / L$ ratio $>2.5$ as compared to patients with N/L ratio $<2.5$ where 6 months PFS was $14 \%$ for patients with N/L ratio $<2.5$ while it was $11 \%$ for patients with N/L ratio $>2.5($ P-value $=0.001)($ Table 2$)$.

2) The following parameters significantly influenced 6 months PFS in a model formed of Gender, N/L ratio, age, platelets:

Gender: (P-value $=0.045)$.

$\mathrm{N} / \mathrm{L}$ ratio: OR: $(\mathrm{P}$-value $=0.045)$.

3) The following parameters significantly influenced one year PFS in a model formed of gender, N/L ratio, age, platelets:

Gender: (P-value: 0.037).

$\mathrm{N} / \mathrm{L}$ ratio: $(\mathrm{P}$-value $=0.042)$.

OS:

There was no statistically significant difference in OS based on N/L ratio where six months OS was $66 \%$ for patients with N/L ratio $<2.5$ while it was $72 \%$ for patients with $\mathrm{N} / \mathrm{L}$ ratio $>2.5(\mathrm{P}$-value $=0.4$ ) (Table 2$)$.

\section{Discussion}

Malignant pleural mesothelioma is a disease of dull prognosis with very slow progress in its management during the past decades so it is important to try to understand its nature and the underlying mechanisms carrying either prognostic or therapeutic value. That is why this study was designed to assess the prognostic value of neutrophil lymphocyte ratio in second line advanced malignant pleural mesothelioma. Our study showed a statistically significant lower 6 months PFS for patients with N/L ratio $>2.5$ versus patients with $\mathrm{N} / \mathrm{L}$ ratio $<2.5$ (P-value = 0.001 ) where there was no significant difference regarding clinical response between the two groups which may 
Table 1. Patients' characteristics.

\begin{tabular}{|c|c|c|}
\hline Character & $\begin{array}{c}\mathrm{N} / \mathrm{L} \text { ratio }>2.5(20 \mathrm{pts}) \\
\text { Number }(\%)\end{array}$ & $\begin{array}{c}\text { N/L ratio }<2.5(30 \mathrm{pts}) \\
\text { Number }(\%)\end{array}$ \\
\hline \multicolumn{3}{|l|}{ Age: } \\
\hline \multicolumn{3}{|l|}{ Sex: } \\
\hline Male & $11(55 \%)$ & 21 (70\%) \\
\hline Female & $9(45 \%)$ & $9(30 \%)$ \\
\hline \multicolumn{3}{|l|}{ Smoking: } \\
\hline Yes & $10(50 \%)$ & $18(60 \%)$ \\
\hline No & $10(50 \%)$ & $12(40 \%)$ \\
\hline \multicolumn{3}{|c|}{ Asbestos exposure } \\
\hline Yes & $14(70 \%)$ & $24(80 \%)$ \\
\hline No & $6(30 \%)$ & $6(20 \%)$ \\
\hline \multicolumn{3}{|l|}{ Histology: } \\
\hline Epithelioid & 18 (90\%) & 27 (90\%) \\
\hline Sarcomatoid & $1(5 \%)$ & $1(3.3 \%)$ \\
\hline Biphasic & $1(5 \%)$ & $2(6.7 \%)$ \\
\hline \multicolumn{3}{|l|}{ ECOG PS: } \\
\hline 1 & $20(100 \%)$ & 29 (96.7\%) \\
\hline 2 & $0(0 \%)$ & $1(3.3 \%)$ \\
\hline \multicolumn{3}{|l|}{ Stage: } \\
\hline III & $6(30 \%)$ & $11(36.7 \%)$ \\
\hline IV & $14(70 \%)$ & 19 (63.3\%) \\
\hline \multicolumn{3}{|c|}{ Type of chemotherapy: } \\
\hline Vinorelbine & 18 (90\%) & 17 (56.7\%) \\
\hline Vorinostat & $2(10 \%)$ & $13(43.3 \%)$ \\
\hline
\end{tabular}

Data were missing for 2 patients.

Table 2. Correlation between N/L ratio and clinical outcome.

\begin{tabular}{cccc}
\hline & PD & $\mathbf{6}$ ms PFS & $\mathbf{6}$ ms OS \\
\hline N/L ratio $>2.5$ & $87.5 \%$ & $11 \%$ & $72 \%$ \\
N/L ratio $<2.5$ & $91.7 \%$ & $14 \%$ & $66 \%$ \\
P-value & 0.66 & 0.001 & 0.4 \\
\hline
\end{tabular}

be attributed to the heterogeneous systemic treatment protocols used. The positive prognostic value of N/L ratio found in this study is in concordance with previous studies either in Mesothelioma or other malignancies like lung cancer, colorectal cancer, and resected intrahepatic cholangiocarcinoma reported by Kao et al., 2010; Sarraf, K.M. et al., 2009; Walsh, S.R. et al. 2005; Kishi, Y. et al. 2009; Halazun, K.J. et al., 2008; Gomez, D. et al., 2008 [10]-[15] whereas the result of this study was contradictory to that reported by Meniawy et al., 2013 [16]. Although the study did not show a significant difference in overall survival based on neutrophil lymphocyte ratio however this may be explained by the relatively small sample size and the nature of the study population being in the second line advanced malignant pleural mesothelioma where it is difficult to elicit survival difference in this settings and so PFS may be a more realistic endpoint and this study showed a significant difference in PFS. The study supports the use of neutrophil lymphocyte ratio as a simple, cheap, widely available and practical prognostic factor in second line advanced malignant pleural mesothelioma after prospective validation. To our knowledge this is the first study assessing the Prognostic value of neutrophil lymphocyte ratio with inclusion of only patients scheduled to second line advanced malignant pleural mesothelioma. One limitation of our study was the relatively small sample size which could not allow assessment of the predictive value of normalization of neutrophil lymphocyte ratio after one cycle of chemotherapy which was addressed in studies done by Kao et al., 2010, Pinato et al., 2014 [10] [17]. One of the values of this thesis is that it supports the knowledge about the 
involvement of inflammatory processes in development of malignant pleural mesothelioma which may have not only prognostic value but also therapeutic value as it may open the way for research into discovery of new drugs targeting any point in the inflammatory pathway including inflammatory mediators.

\section{Conclusion}

In our study, it was concluded that N/L ratio is a potential prognostic marker for advanced MPM treated with the second line chemotherapy which deserves validation by prospective studies including a larger number of patients, furthermore it may have a therapeutic implication through the possible targeting of inflammatory pathway in malignant pleural mesothelioma.

\section{Acknowledgements}

- This work was presented as a poster at the 15th World Conference on Lung Cancer (WCLC 2013) in Sydney, Australia from October 27-October 30, 2013.

- The authors thank Dr. Tarneem Mahmoud for preparation of the statistics of this study.

\section{References}

[1] Price, B. and Ware, A. (2009) Time Trend of Mesothelioma Incidence in the United States and Projection of Future Cases: An Update Based on SEER Data for 1973 through 2005. Critical Reviews in Toxicology, 39, 576-588. http://dx.doi.org/10.1080/10408440903044928

[2] Pelucchi, C., Malvezzi, M., La Vecchia, C., et al. (2004) The Mesothelioma Epidemic in Western Europe: An Update. British Journal of Cancer, 90, 1022-1024. http://dx.doi.org/10.1038/sj.bjc.6601638

[3] Curran, D., Sahmoud, T., Therasse, P., van Meerbeeck, J., Postmus, P.E. and Giaccone, G. (1998) Prognostic Factors in Patients with Pleural Mesothelioma: The European Organization for Research and Treatment of Cancer Experience. Journal of Clinical Oncology, 16, 145-152.

[4] Herndon, J.E., Green, M.R., Chahinian, A.P., Corson, J.M., Suzuki, Y. and Vogelzang, N.J. (1998) Factors Predictive of Survival among 337 Patients with Mesothelioma Treated between 1984 and 1994 by the Cancer and Leukemia Group B. Chest, 113, 723-731. http://dx.doi.org/10.1378/chest.113.3.723

[5] Wang, X. (2014) Neutrophil to Lymphocyte Ratio in Relation to Risk of All-Cause Mortality and Cardiovascular Events among Patients Undergoing Angiography or Cardiac Revascularization: A Meta-Analysis of Observational Studies. Atherosclerosis, 234, 206-213. http://dx.doi.org/10.1016/j.atherosclerosis.2014.03.003

[6] Wang, J. (2014) The Clinical Significance of Tumor-Infiltrating Neutrophils and Neutrophil-to-CD8+ Lymphocyte Ratio in Patients with Resectable Esophageal Squamous Cell Carcinoma. Journal of Translational Medicine, 12, 7. http://dx.doi.org/10.1186/1479-5876-12-7

[7] Xue, P. (2014) Neutrophil-to-Lymphocyte Ratio for Predicting Palliative Chemotherapy Outcomes in Advanced Pancreatic Cancer Patients. Cancer Medicine, 3, 406-415. http://dx.doi.org/10.1002/cam4.204

[8] Jarnicki, A., Putoczki, T. and Ernst, M. (2010) Stat3: Linking Inflammation to Epithelial Cancer-More than a "Gut" Felling? Cell Division, 5, 14. http://dx.doi.org/10.1186/1747-1028-5-14

[9] Byrne, M.J. and Nowak, A.K. (2004) Modified RECIST Criteria for Assessment of Response in Malignant Pleural Mesothelioma. Annals of Oncology, 15, 257-260. http://dx.doi.org/10.1093/annonc/mdh059

[10] Kao, S., Pavlakis, N., Harvie, R., Vardy, J., Boyer, M., van Zandwijk, N. and Clarke, S. (2010) High Blood Neutrophilto-Lymphocyte Ratio Is an Indicator of Poor Prognosis in Malignant Mesothelioma Patients Undergoing Systemic Therapy. Clinical Cancer Research, 16, 5805-5813. http://dx.doi.org/10.1158/1078-0432.CCR-10-2245

[11] Sarraf, K.M., Belcher, E., Raevsky, F., Nicholson, A.G., Goldstraw, P. and Lim, E. (2009) Neutrophil/Lymphocyte Ratio and Its Association with Survival after Complete Resection in Non-Small Cell Lung Cancer. Journal of Thoracic and Cardiovascular Surgery, 137, 425-428. http://dx.doi.org/10.1016/j.jtcvs.2008.05.046

[12] Walsh, S.R., Cook, E.J., Goulder, F., Justin, T.A. and Keeling, N.J. (2005) Neutrophil-Lymphocyte Ratio as a Prognostic Factor in Colorectal Cancer. Journal of Surgical Oncology, 91, 181-184. http://dx.doi.org/10.1002/jso.20329

[13] Kishi, Y., Kopetz, S., Chun, Y., Palavecino, M., Abdalla, E.K. and Vauthey, J.N. (2009) Blood Neutrophil-to-Lymphocyte Ratio Predicts Survival in Patients with Colorectal Liver Metastases Treated with Systemic Chemotherapy. Annals of Surgical Oncology, 16, 614-622. http://dx.doi.org/10.1245/s10434-008-0267-6

[14] Halazun, K.J., Aldoori, A., Malik, H.Z., Al-Mukhtar, A., Prasad, K.R., Toogood, G.J., et al. (2008) Elevated Preoperative Neutrophil to Lymphocyte Ratio Predicts Survival Following Hepatic Resection for Colorectal Liver Metastases. 
European Journal of Surgical Oncology, 34, 55-60. http://dx.doi.org/10.1016/j.ejso.2007.02.014

[15] Gomez, D., Morris-Stiff, G., Toogood, G., Lodge, J.P. and Prasad, K.R. (2008) Impact of Systemic Inflammation on Outcome Following Resection for Intrahepatic Cholangiocarcinoma. Journal of Surgical Oncology, 97, 513-518. http://dx.doi.org/10.1002/jso.21001

[16] Meniawy, T., Creaney, J., Lake, R. and Nowak, A. (2013) Existing Models, but Not Neutrophil-to-Lymphocyte Ratio, Are Prognostic in Malignant Mesothelioma. British Journal of Cancer, 109, 1813-1820. http://dx.doi.org/10.1038/bjc.2013.504

[17] Pinato, D., Stavraka, C., Flynn, M., Forster, M., O’Cathail, S., Seckl, M., Kristeleit, R., Olmos, D., Turnbull, S. and Blagden, S. (2014) An Inflammation Based Score Can Optimize the Selection of Patients with Advanced Cancer Considered for Early Phase Clinical Trials. PLoS ONE, 9, Article ID: e83279.

http://dx.doi.org/10.1371/journal.pone.0083279 